\title{
Anticipation-specific Reliability and Trial- to-Trial Carryover of Anticipatory Attentional Bias for Threat
}

\author{
Thomas E. Gladwin ${ }^{a^{*}}$
}

\author{
Bernd Figner ${ }^{\text {bc }}$ \\ Matthijs Vink de
}

${ }^{a}$ Department of Psychology \& Counselling, University of Chichester. College Lane,

Chichester, PO19 6PE, United Kingdom. Email: thomas.gladwin@ gmail.com. Tel.:

$+44(0) 7895625183$.

${ }^{\mathrm{b}}$ Behavioural Science Institute, Radboud University Nijmegen, Nijmegen, The Netherlands

${ }^{c}$ Donders Centre for Cognitive Neuroimaging, Nijmegen, The Netherlands

${ }^{\mathrm{d}}$ Department of Psychiatry, Brain Center Rudolf Magnus, Utrecht University Medical

Center, Utrecht, The Netherlands

${ }^{\mathrm{e}}$ Departments of Developmental and Experimental Psychology, Utrecht University, Utrecht, The Netherlands

* Corresponding author. 
Anticipatory attentional bias

\section{Abstract}

Concerns have been raised about the reliability of the widely-used dot-probe task. A novel variation, the cued Visual Probe Task (cVPT), uses cues that predict the locations of emotional stimuli. This appears to result in relatively reliable anticipatory attentional bias scores. However, cVPT reliability could be affected by individuals having an attentional bias towards one or the other predictive cue, rather than to predicted stimuli. We provide an improved assessment of this bias. Further, trial-to-trial carryover effects, a source of withinsubject attentional bias variability previously found for stimulus-evoked biases, were tested. To these aims, a convenience sample of 82 participants was analysed, who performed an online procedure including a reversal of the cue mapping of the cVPT. The predicted stimulus categories were neutral and angry faces. CSIs of 400 and $1000 \mathrm{~ms}$ were used based on previous work. An overall anticipatory attentional bias, in terms of RT difference scores, towards threat was found. Reliability was around .4 , which was similar to previous results despite the cue reversal procedure. Carryover effects were found with a similar pattern as for non-cued threat-evoked bias. The results confirm a reasonably reliable outcome-focused bias towards threat, showing similar carryover effects as found for stimulus-evoked bias.

\section{Keywords}

Cued Visual Probe Task; anticipatory attentional bias; reliability; threat; carryover. 
Anticipatory attentional bias

The dot-probe task (MacLeod, Mathews, \& Tata, 1986; Mogg \& Bradley, 1999) has

frequently been used as a measure of attentional bias towards or away from hypothetically salient stimuli, such as threat or alcohol. Such biases have been suggested to be related to a wide range of mental health problems. For example, a bias towards threatening stimuli may be related to anxiety (Bantin, Stevens, Gerlach, \& Hermann, 2016; Cisler \& Koster, 2010) although gain results are complex, involving increased bias towards threat but also attentional avoidance and reduced ability to disengage from threatening stimuli. Similarly, a bias towards alcohol has been associated with heavier drinking (Field, Mogg, Zetteler, \& Bradley, 2004; Townshend \& Duka, 2001), but also in this domain results are not straightforward, some studies indicating that whether attention is directed towards or away from alcohol is strongly dependent on the precise cue-stimulus interval, i.e., the interval between cue and probe stimulus presentation (Noël et al., 2006; Townshend \& Duka, 2007; Vollstädt-Klein, Loeber, von der Goltz, Mann, \& Kiefer, 2009). An important issue with the dot-probe task is that it has been found to have unacceptable reliability, as has been extensively discussed elsewhere (Ataya et al., 2012; Brown et al., 2014; Chapman, Devue, \& Grimshaw, 2019; Christiansen, Schoenmakers, \& Field, 2015; Dear, Sharpe, Nicholas, \& Refshauge, 2011; Kappenman, Farrens, Luck, \& Proudfit, 2014; McNally, 2018; Puls \& Rothermund, 2018; Schmukle, 2005; Waechter, Nelson, Wright, Hyatt, \& Oakman, 2014). In some reported cases internal reliability has been found to be close to zero, e.g., in a dot-probe task using emotional faces (Brown et al., 2014), angry and neutral faces, especially at longer cuestimulus intervals (Chapman et al., 2019), personalized pain-related stimuli (Dear et al., 2011), and, for some conditions, alcoholic stimuli (Ataya et al., 2012). Low reliability is a fundamental problem for lines of research that focus on relationships between individual differences in the attentional bias and, e.g., symptoms. In simplified terms, if a measure cannot predict itself, it cannot predict anything else, and any statistical findings involving 
Anticipatory attentional bias

correlational analyses would therefore likely reflect noise and false positives. Note that this does not affect the ability of a measure to reveal strong within-subject effects or betweengroup differences if the lack of reliability concerns only within-group variation (MacLeod, Grafton, \& Notebaert, 2019). Further, it is conceivable that complex trial-to-trial changes in psychological state could lead to a measure in which validity and reliability, measured via split-half reliability, are somewhat disconnected, in contradiction to simplistic conceptualizations of the reliability-validity relationship. Consider, as an extreme toy example, a task with two trials, only the first of which reflects an individual difference strongly correlated with, e.g., anxiety; the second trial, however, only reflects a floor or ceiling effect for all participants, perhaps because the stimuli used habituate strongly. The split-half correlation between the two trials could be zero, while the score calculated over both trials could be highly correlated with anxiety - thus, the measure would be unreliable but valid. This would appear to follow, in a general sense, from it being an unrealistic assumption that individual trials of an implicit measure can be considered as equivalent to each other as could be expected from items in a questionnaire's subscale.

$<$ Figure 1 around here>

Nevertheless, it could be desirable to simply have an attentional bias measure with good reliability. One approach related to this issue has been the development of the cued Visual Probe Task, cVPT (Gladwin, 2016; Gladwin \& Vink, 2018). In the cVPT (Figure 1), visually neutral cues predict the occurrence of emotionally salient stimuli versus control stimuli at the cues' locations. These stimuli actually occur on only half the trials; on the other half, probes appear requiring a response. The probes consist of a target stimulus and a distractor stimulus, and participants are required to press a button corresponding to the location of the target stimulus. The cVPT can be contrasted with the use of previously conditioned cues in a spatial attention task (Koster, Crombez, Van Damme, Verschuere, \& De Houwer, 2005; Le Pelley, 
Vadillo, \& Luque, 2013; Notebaert, Crombez, Van Damme, De Houwer, \& Theeuwes, 2011). For instance, an initially neutral cue could be associated with an addictive substance, and that cue can subsequently be shown to draw attention as measured by a traditional dot-probe task (Field \& Duka, 2002; Hogarth \& Duka, 2006). The concept of an anticipatory attentional bias is somewhat different from a conditioned cue-evoked attentional bias, although we note that the underlying mechanisms could well overlap. The idea that predictive processes could induce an automatic anticipatory bias was based on the $\mathrm{R}^{3}$ model (Gladwin \& Figner, 2014; Gladwin, Figner, Crone, \& Wiers, 2011): One aspect of this model is that automatic biases can involve processes predicting emotionally salient outcomes, rather than only stimulusdriven processes. Such "low-level" predictive processes are a fundamental brain function, served by neural information processing in the basal ganglia (Ernst et al., 2004; Hollerman, Tremblay, \& Schultz, 2000; Samejima \& Doya, 2007). Predicted outcomes would be expected to bias the selection of responses towards those with better predicted outcomes, including covert cognitive responses such as attentional shifts. Evidence that this outcomefocused automatic process does indeed underlie the anticipatory bias was provided by a training study: training participants to direct attention towards versus away from a cued salient stimulus category, using a predictive form of Attentional Bias Modification, was found to result in a subsequent stimulus-evoked bias (Gladwin, Möbius, \& Becker, 2019). That is, performing a training version of the $\mathrm{cVPT}$ affected processes related to the predicted stimulus categories, and not just the conditioned cues. Because performance on the cVPT is measured on trials in which no exemplars of the emotional and control stimuli are presented, the influence of low-level visual confounds is minimized (Gray, Adams, \& Garner, 2010; Gray, Adams, Hedger, Newton, \& Garner, 2013) and trial-to-trial noise in the bias due to the particular exemplars of the emotional and control stimuli used on particular trials is removed. Accordingly, bias scores have indeed been found to have improved split-half reliability: the 
cued bias for threat stimuli had higher reliability than the stimulus-evoked attentional bias for the same stimuli (Gladwin, Möbius, Mcloughlin, \& Tyndall, 2019) and very good reliability has been found for alcohol stimuli (Gladwin, 2019).

However, an issue arises when applying the usual assessment of split-half reliability to the cVPT. This is due to the fact that, unlike the dot-probe task in which there are only the salient versus control cues, in the cVPT there are both the predicted stimulus categories and the visually distinct cues. It is conceivable that there are individual differences in attentional biases involving the cues. This does not threaten the validity of tests of within-subject anticipatory effects: the mapping from cues to predicted stimulus categories is randomized per participant. However, note that the essential issue is that an individual could, for instance, have a preference for blue versus yellow cues, or for X's versus O's, and the counterbalancing of cue-mapping would do nothing to avoid the effect of such preferences on reliability. There is thus uncertainty whether previous findings on reliability truly reflect the individual differences of interest, namely differences in anticipatory attentional bias involving the predicted stimulus categories, i.e., the cue-contingent outcome of performing attentional shifts. The primary aim of the current study was to find and apply a method to address this issue of assessing reliability for cued attentional bias tasks.

A second aim of the study was to replicate and generalize the finding of carryover effects on attentional biases (Gladwin, 2017a; Gladwin \& Figner, 2019; Hill \& Duval, 2016). "Carryover" here refers to the dependence of the attentional bias on the location of the probe on the previous trial: is the attentional bias towards a certain stimulus category measured on trial $N$ different when the probe stimulus on the previous trial $N-1$ was presented at the location of the same or the other stimulus category? Various theoretical concepts could be used to attempt to understand such a phenomenon, if it is found to exist. For example, the attentional bias could be seen as a task set (Monsell, 2003) of stimulus-response mappings, in 
Anticipatory attentional bias

which the response is an attentional shift; perhaps responding to a probe at a given location establishes such a task set which could then influence the subsequent trial; or there could be a form of binding (Roelfsema, Engel, König, \& Singer, 1997) between the probe location's stimulus category and an attentional function; the estimated probability of probe locations could be affected (Failing \& Theeuwes, 2018); or the experience of correctly responding to the probe could serve to reinforce the shift of attention to the category associated with the probe's location (Chelazzi et al., 2014; Della Libera \& Chelazzi, 2009). Whatever the mechanism, previous results indicate the existence of strong carryover effects. For simple color stimuli, responding to a probe presented at the location of one of the colors leads to an attentional bias towards that color on the next trial; and for threat versus control stimuli, it was found that an attentional bias towards threat occurs only following trials in which the probe was presented at the threat location (Gladwin \& Figner, 2019). Such effects are potentially important as a source of within-subject attentional bias variability, which has received recent research interest (Gladwin \& Vink, 2018; Iacoviello et al., 2014; Kruijt et al., 2016; Swick \& Ashley, 2017; Zvielli, Bernstein, \& Koster, 2014). However, previous carryover studies concerned the usual stimulus-evoked biases, rather than cue-evoked biases, and it is as yet unknown in what way carryover would occur in the cVPT. The current study therefore aimed to determine whether carryover effects would occur on the anticipatory attentional bias.

Thus, to address the first aim of the study, the cVPT procedure was adjusted to evaluate the reliability of the anticipatory attentional bias for threat while controlling for the possible influence of cue-related biases. All participants performed two versions all the cVPT, with reversed mappings from cues to predicted emotional stimuli. We hypothesized that the splithalf reliability over the whole task would remain similar to the reliability previously found when a constant within-subject cue-outcome mapping was used. This would confirm that 
Anticipatory attentional bias

there are indeed stable individual differences in outcome-focused rather than cue-related biases, that can be measured using the cVPT. Further, the data were used to test whether the previous findings of carryover effects would generalize to the anticipatory attentional bias. Finally, the study was an opportunity to replicate the overall effect of an anticipatory bias towards threat.

\section{Methods}

\section{Participants}

The experiment was completed online by an analytical sample of 82 healthy adult participants ( 44 female, 38 male; age $30, S D=10$ ). A further 21 participants performed the experiment but were excluded in quality checks for low accuracy (mean accuracy below .90, $n=2$ ), incorrect report of the cue - stimulus mapping on essential time points (see Procedure; $n=16$ ), an extreme (i.e., $\mid z$-score $\mid>3$ ) bias $(n=3)$ or an extreme RT (but $n=0$ ). Such quality checks were considered important given the increased uncertainty of the compliance of participants in online studies; however, we briefly note that when analyses were repeated for the full sample the results were very similar, except for a lower reliability for the $400 \mathrm{~ms}$ CSI. All participants gave informed consent and the study was conducted following institutional ethical procedures.

\section{cVPT}

The cVPT was programmed in JavaScript, based on open source code for a general online attentional bias task (Gladwin, 2017b), with data being saved using AJAX requests to a PHP script on the server. Trials on the cVPT were divided into Picture and Probe trials; whether a trial was a Picture or Probe trial was randomly selected per trial. Probe trials began with a central fixation cross for 150,200 , or 250 ms. This was followed by two visually neutral cues: the symbols 00000 and XXXXX, presented in yellow (RGB values 250, 250, 10) versus light 
Anticipatory attentional bias

blue (RGB values $10,250,250)$. While cue symbols and colors were somewhat arbitrary, they were chosen to be clearly visually distinct and to not have very obvious emotional associations. Cues subtended around 2 degrees visual angle vertically and 5 degrees horizontally, although this could not be precisely controlled due to variations in participants' computer screens. There were no restrictions on display size or type, beyond a laptop or desktop being required to perform the task rather than phone or tablet. The cues were onscreen for a Cue-Stimulus Interval (CSI) of 400 or 1000 ms. These CSIs were previously found to have reasonable reliability for a similar cVPT (Gladwin, Möbius, Mcloughlin, et al., 2019). The cues were located on one of the diagonals, alternating per trial: either on the topleft and bottom-right, or on the bottom-left and top-right. After the cue, a probe stimulus, $\gg><$, was presented at one of the cue locations, and a distractor stimulus, $M$ or $\backslash$, at the other location. Cue subtended around 2 degrees vertically and 4 degrees horizontally. There was a $5 \%$ chance of a trial being a catch trial, on which there were two distractors and no target; this was done to reduce the chance of participants responding to distractors, which in principle would imply that the target was at the opposite location of the diagonal, rather than seeking out the target. The probe stimulus was presented for $1000 \mathrm{~ms}$, or until a response was given if faster than 1000 ms. The task was to quickly and accurately press a key (R for top-left, F for bottom-left, J for bottom-right or I for top-right) corresponding to the probe location. Fingers were instructed to be placed on the keys so that the spatial congruence between probe and key positions was clear, i.e., left and right index fingers on the $\mathrm{F}$ and $\mathrm{J}$ keys, respectively and left and right middle fingers on the R and I keys, respectively. Errors were followed by a red "Incorrect!" for incorrect responses, and a red "Too late!" if no response was given, for 200 ms. 
Anticipatory attentional bias

On Picture trials, instead of a probe stimulus, pictures were presented, one at each of the cue locations. One of the cues was always replaced by an angry face (the Threat category) from the BESST (Thoma, Soria Bauser, \& Suchan, 2013) and the other cue was always replaced by an neutral face (the Neutral category). Each picture subtended around 9degrees horizontally and 13 degrees vertically. The pictures remained onscreen for $1000 \mathrm{ms,}$ followed by 200 ms of empty screen.

\section{Procedure}

Participants performed the experiment fully online. There were two phases. In the first phase, one of the two possible mappings from predictive cues to stimulus category was chosen at random per participant. With this mapping kept constant within the first phase, the participant performed two short training runs (two blocks of 24 trials) and one assessment run ( 8 blocks of 24 trials). After each run an awareness check was performed, in which participants were asked which of the cues predicted the location of angry and of neutral faces. The second phase followed on immediately and was identical to the first phase, but the cue - stimulus mapping was reversed. This led to six awareness checks. The essential checks for quality control were checks $2,3,5$ and 6 : that is, participants were required to be aware of the mapping from start to finish of the assessment block. Due to this reversal of cue-outcome mapping from the first to the second phase, any attentional preference involving cue stimuli would be cancelled out and could not affect the reliability of the threat-related attentional bias. For completeness we note that varying sets of questionnaires related to mental health were presented at the start of the task, solely for exploratory purposes; these are presented for transparency in Supplementary Materials. 
Anticipatory attentional bias

\section{Preprocessing and statistical analyses}

Per participant, trials were removed that were likely not to reflect normal task performance: the first four trials of each task, due to possible start-up effects following a break and (re)introduction to the task; trials with a very fast response ( $<50 \mathrm{~ms})$ which were too fast to reflect a true response as opposed to a fast guess; trials following an error, which could be affected by the error feedback or realization of the error, e.g., post-error slowing; the first trial of each block, as these followed a brief break rather than a preceding trial as all subsequent trials of the block; and trials with an RT more than 3 SD away from the mean of the experimental condition the trial was in, which could reflect an abnormal situation in which the participant was insufficiently engaged in the task. Of the remaining probe trials, the median reaction time was calculated for the Probe-on-Threat and Probe-on-Neutral predictive cue locations, for each CSI, per participant.

Data were processed and analysed using custom software in Matlab (The Mathworks, 2015). Repeated measures ANOVA was used to test within-subject effects on probe location, previous probe location (i.e., the location of the most recent probe stimulus, used to test for carryover effects) and CSI. The dependent variable was the median RT per participant, with effects involving attentional bias being tested via the factor probe location. The reliability of the bias, i.e., the contrast between the median RT difference between the Threat versus Neutral location, was tested using the Spearman-Brown formula for the split-half reliability of the task (using Spearman's correlations), divided into sets of even and odd numbered blocks. Further, because misleadingly high or otherwise non-representative correlations can be easily caused by influential data points, the following procedure was used to reduce such possible effects on reliability (scatterplots were also visually inspected for any conspicuous issues). The change in correlation caused by removing a data point was calculated for all 
Anticipatory attentional bias

data points. If removal of a data point resulted in an absolute change with a z-score above 3, that data point was removed from the calculation of the reliability. This resulted in the removal of either one (for the overall bias and the bias at $1000 \mathrm{~ms}$ CSI) or two (for the bias at $400 \mathrm{~ms}$ CSI) data points.

Data and software will be made available upon request.

\section{Results}

RT and accuracy data are shown in Figure 2 and Table 1.

$<$ Figure 2>

$<$ Table 1>

For $\mathrm{RT}$, responses to probes at the Threat versus Neutral location were faster, $F(1,81)=$ $12.72, p<.001, \eta_{p}{ }^{2}=0.14$. The bias was dependent on a carryover effect as shown by an interaction between probe location and previous probe location, $F(1,81)=5.35, p=0.023$, $\eta_{p}{ }^{2}=0.062$. This carryover interaction was explored via tests of probe location separately for each level of previous probe location. When the previous probe location was on the predicted Threat location, a bias towards threat was found, $F(1,81)=17.71, p<.001, \eta_{p}^{2}=$ 0.18. This bias was not found when the previous probe location was on the predicted Neutral location, $F(1,81)=1.09, p=.30, \eta_{p}{ }^{2}=0.013$. Thus, the bias towards threat only occurred following trials when the probe had been presented on the Threat location. Responses at the $1000 \mathrm{~ms}$ CSI were faster than at the $400 \mathrm{~ms} \operatorname{CSI}, F(1,81)=4.97, p=.029$, $\eta_{p}{ }^{2}=0.058$. All other effects were non-significant: previous probe location, $F(1,81)=1.94, p$ $=.17, \eta_{p}^{2}=0.023$; previous probe location $\times$ CSI, $F(1,81)=2.60, p=.11, \eta_{p}^{2}=0.031$; and probe location $x$ previous probe location $\times$ CSI, $F(1,81)=0.00, p=.99, \eta_{p}{ }^{2}=0.00$. 
Anticipatory attentional bias

The reliability of RT-based bias scores was .35 overall, .41 for 400 ms CSI and .45 for 1000 ms CSI.

We briefly note for completeness that no significant effects were found on accuracy.

\section{Discussion}

The current study aimed to provide an improved test of the reliability of the anticipatory attentional bias and to replicate the previous finding of an overall anticipatory bias towards threat. The primary result was that split-half reliability of the anticipatory attentional bias for threat remained similar to a previous study (Gladwin, Möbius, Mcloughlin, et al., 2019), even though the specific cues predicting outcomes of attentional shifting were reversed on half the trials. This supports the interpretation of the reliability in terms of an individual difference in bias towards the predicted emotional stimulus categories, rather than bias involving the visual features of the predictive cues. Further, an overall bias towards threat found in the previous study was replicated, involving a roughly $10 \mathrm{~ms}$ difference as seen in Figure 1 and Table 1. Further, a hypothesized generalization of trial-to-trial carryover effects was confirmed: the bias towards threat was dependent on the most recent previous probe having been presented at the threat location.

The reliability of the anticipatory attentional bias for threat thus appears to be robust, although modest, and not dependent on individual differences involving cue characteristics. Higher reliability has been found for the anticipatory attentional bias for alcohol (Gladwin, 2019), which may reflect a wider range of individual differences in bias in the sampled population. While the majority of individuals may show a general bias towards threat, and it appears that these are at least to some extent a stable individual difference, there would be expected to be more differentiation involving alcohol involving a range of personal factors 
Anticipatory attentional bias

such as experience with drinking and the consequences of alcohol use (Field et al., 2004). Further, it remains possible that group differences in the threat-related bias between healthy controls and clinical populations could be strong, as such differences would not depend on a high reliability within the healthy population. However, given the current and previous findings, future work on individual differences should consider using a longer version of the cVPT to increase reliability.

The predicted carryover effects were found, generalizing the previous findings on trial-totrial carryover in stimulus-evoked attentional threat bias (Gladwin \& Figner, 2019) to the anticipatory attentional bias. Such effects are potentially methodologically important for studies of attentional bias variability, ABV (Gladwin \& Vink, 2018; lacoviello et al., 2014; Zvielli et al., 2014), as carryover would seem likely to explain at least some such withinsubject variability: If a simple bias occurs that is, however, present following one trial type but not another, this would naturally lead to variability. It could thus be important to assess ABV separately for trials following different trial types. The current finding of carryover further implies that whether an attentional bias is found on a given trial is determined by a state, induced by the previous probe's location, that can occur quickly and flexibly on a trialto-trial basis. This state could be described in various theoretical terms, such as the reinforcement of cognitive actions (Gladwin et al., 2011), the creation of an event file (Hommel, 2004; Lavender \& Hommel, 2007), an attentional set or task set involving internal, cognitive responses (Monsell, 2003; Rushworth, Passingham, \& Nobre, 2005), or the binding of actions to stimuli (Roelfsema et al., 1997; Singer et al., 1996). It remains, however, to be determined what the best specific model is of the mechanisms underlying carryover effects. However, it may be informative that the cVPT's carryover shares an asymmetry with the stimulus-evoked carryover for threat stimuli: in both cases, a bias towards threat occurs 
Anticipatory attentional bias

only following a probe-on-threat trial. The binding or task set must thus involve predicted stimulus categories, rather the visual features of the cues. This asymmetry also suggests that the carryover was not due to statistical learning of the likely probe location, as this would be equal following probe-on-threat and probe-on-neutral trials. Further, the current result shows that carryover can occur without particular exemplars from stimulus categories.

The current study was limited in its use of a convenience sample measured online. Although it has been noted that online data do not appear to be radically different from laboratory data (Chetverikov \& Upravitelev, 2016), noisier data would seem likely despite quality checks. Laboratory-based study of the cVPT could conceivably reveal improved reliability. However, it is notable in this regard that it was also an online study that found high reliability for an alcohol-related attentional bias. This suggests that it is the nature of stimulus categories that determines reliability rather than whether the data were acquired online. Further, the current data do not allow detailed claims to be made on underlying processes. While there is evidence for some form of anticipatory bias, whether this relates to early selection of information or later processes transforming probes to responses is unknown. We do note that this uncertainty is not unique to the currently used target detection task; if the task had involved a speeded choice as common in dot-probe tasks, there could still have been, e.g., inhibitory or disinhibitory effects at the level of response execution (Mogg, Holmes, Garner, \& Bradley, 2008). Answering questions at this lower level of description would seem to require, ideally, psychophysiological measurements measuring neural activity during preparatory intervals (Alilović, Timmermans, Reteig, van Gaal, \& Slagter, 2019; Bastiaansen, Böcker, Brunia, de Munck, \& Spekreijse, 2001; Bastiaansen, 
Anticipatory attentional bias

Posthuma, Groot, \& de Geus, 2002; Bastiaansen \& Brunia, 2001; Gladwin \& de Jong, 2005; Korucuoglu, Gladwin, \& Wiers, 2014; Pourtois \& Vuilleumier, 2006; Slagter, Alilovic, \& Van Gaal, 2018). The current study was further limited to a particular set of cues, distinguished on both colour and form. It remains to be determined under which conditions cues are able to evoke an anticipatory attentional bias, and which features would be optimal for this. Finally, a limitation of the study is that CSIs of 400 and 1000 ms were used. The current data cannot determine whether, e.g., the presence of the 1000 ms CSI was necessary for participants to learn the contingency between cues and threat-related outcomes and hence for effects to occur on either CSI.

In conclusion, an improved assessment of the reliability of the anticipatory attentional bias for threat was performed. Similar levels of split-half reliability were found, that could no longer be explained by stable biases involving basic visual features of cues. Further, an overall anticipatory bias towards threat as well as trial-to-trial carryover effects were replicated. The results thus provide further support for the theoretical construct of outcome-focused automatic attentional processes and suggest potentially important directions for future assessment methods and analyses. Given the current results, future work would seem justified aimed at testing relationships between the anticipatory attentional bias and individual differences, e.g., in mental health.

\section{References}

Alilović, J., Timmermans, B., Reteig, L. C., van Gaal, S., \& Slagter, H. A. (2019). No Evidence that Predictions and Attention Modulate the First Feedforward Sweep of Cortical Information Processing. Cerebral Cortex, 29(5), 2261-2278. https://doi.org/10.1093/cercor/bhz038 
Anticipatory attentional bias

Ataya, A. F., Adams, S., Mullings, E., Cooper, R. M., Attwood, A. S., \& Munafò, M. R. (2012). Internal reliability of measures of substance-related cognitive bias. Drug and Alcohol Dependence, 121(1-2), 148-151. https://doi.org/10.1016/j.drugalcdep.2011.08.023

Bantin, T., Stevens, S., Gerlach, A. L., \& Hermann, C. (2016). What does the facial dot-probe task tell us about attentional processes in social anxiety? A systematic review. Journal of Behavior Therapy and Experimental Psychiatry, 50. https://doi.org/10.1016/j.jbtep.2015.04.009

Bastiaansen, M. C. M., Böcker, K. B., Brunia, C. H., de Munck, J. C., \& Spekreijse, H. (2001). Event-related desynchronization during anticipatory attention for an upcoming stimulus: A comparative EEG/MEG study. Clinical Neurophysiology : Official Journal of the International Federation of Clinical Neurophysiology, 112(2), $393-403$.

Bastiaansen, M. C. M., \& Brunia, C. H. (2001). Anticipatory attention: An event-related desynchronization approach. International Journal of Psychophysiology : Official Journal of the International Organization of Psychophysiology, 43(1), 91-107.

Bastiaansen, M. C. M., Posthuma, D., Groot, P. F. C., \& de Geus, E. J. C. (2002). Eventrelated alpha and theta responses in a visuo-spatial working memory task. Clinical Neurophysiology: Official Journal of the International Federation of Clinical Neurophysiology, 113(12), 1882-1893.

Brown, H. M., Eley, T. C., Broeren, S., MacLeod, C. M., Rinck, M., Hadwin, J. A., \& Lester, K. J. (2014). Psychometric properties of reaction time based experimental paradigms measuring anxiety-related information-processing biases in children. Journal of Anxiety Disorders, 28(1), 97-107. https://doi.org/10.1016/j.janxdis.2013.11.004 
Anticipatory attentional bias

Chapman, A., Devue, C., \& Grimshaw, G. M. (2019). Fleeting reliability in the dot-probe task. Psychological Research, 83(2), 308-320. https://doi.org/10.1007/s00426-0170947-6

Chelazzi, L., E to inova, J., Calletti, R., Lo Gerfo, E., Sani, I., Della Libera, C., \& Santandrea, E. (2014). Altering Spatial Priority Maps via Reward-Based Learning. Journal of Neuroscience, 34(25), 8594-8604. https://doi.org/10.1523/JNEUROSCI.0277-

\subsection{4}

Chetverikov, A., \& Upravitelev, P. (2016). Online versus offline: The Web as a medium for response time data collection. Behavior Research Methods, 48(3), 1086-1099. https://doi.org/10.3758/s13428-015-0632-x

Christiansen, P., Schoenmakers, T. M., \& Field, M. (2015). Less than meets the eye: Reappraising the clinical relevance of attentional bias in addiction. Addictive Behaviors, 44, 43-50. https://doi.org/10.1016/j.addbeh.2014.10.005

Cisler, J. M., \& Koster, E. H. W. (2010). Mechanisms of attentional biases towards threat in anxiety disorders: An integrative review. Clinical Psychology Review, 30(2), 203216. https://doi.org/10.1016/j.cpr.2009.11.003

Dear, B. F., Sharpe, L., Nicholas, M. K., \& Refshauge, K. (2011). The psychometric properties of the dot-probe paradigm when used in pain-related attentional bias research. The Journal of Pain: Official Journal of the American Pain Society, 12(12), 1247-1254. https://doi.org/10.1016/j.jpain.2011.07.003

Della Libera, C., \& Chelazzi, L. (2009). Learning to attend and to ignore is a matter of gains and losses. Psychological Science : A Journal of the American Psychological Society / APS, 20(6), 778-84. https://doi.org/10.1111/j.1467-9280.2009.02360.x 
Anticipatory attentional bias

Ernst, M., Nelson, E. E., McClure, E. B., Monk, C. S., Munson, S., Eshel, N., ... Pine, D. S. (2004). Choice selection and reward anticipation: An fMRI study. Neuropsychologia, 42(12), 1585-1597. https://doi.org/10.1016/j.neuropsychologia.2004.05.011

Failing, M., \& Theeuwes, J. (2018). Selection history: How reward modulates selectivity of visual attention (Vol. 25). https://doi.org/10.3758/s13423-017-1380-y

Field, M., \& Duka, T. (2002). Cues paired with a low dose of alcohol acquire conditioned incentive properties in social drinkers. Psychopharmacology, 159(3), 325-34. https://doi.org/10.1007/s00213-001-0923-z

Field, M., Mogg, K., Zetteler, J., \& Bradley, B. P. (2004). Attentional biases for alcohol cues in heavy and light social drinkers: The roles of initial orienting and maintained attention. Psychopharmacology, 176(1), 88-93. https://doi.org/10.1007/s00213-004$1855-1$

Gladwin, T. E. (2016). Attentional bias variability and cued attentional bias for alcohol stimuli. Addiction Research and Theory, 25(1), 32-38. https://doi.org/10.1080/16066359.2016.1196674

Gladwin, T. E. (2017a). Carryover effects in spatial attentional bias tasks and their relationship to subclinical PTSD symptoms. Traumatology, 23(4), 303-308. https://doi.org/10.1037/trm0000121

Gladwin, T. E. (2017b). OnlineCBM. https://doi.org/10.5281/zenodo.844038

Gladwin, T. E. (2019). Spatial Anticipatory Attentional Bias for Alcohol: A Preliminary Report on Reliability and Associations with Risky Drinking. Alcoholism and Drug Addiction. https://doi.org/10.31234/OSF.IO/WP6S5

Gladwin, T. E., \& de Jong, R. (2005). Bursts of occipital theta and alpha amplitude preceding alternation and repetition trials in a task-switching experiment. Biological Psychology, 68(3), 309-329. https://doi.org/10.1016/j.biopsycho.2004.06.004 
Anticipatory attentional bias

Gladwin, T. E., \& Figner, B. (2014). "Hot” cognition and dual systems: Introduction, criticisms, and ways forward. In E. Wilhelms \& V. F. Reyna (Eds.), Frontiers of Cognitive Psychology Series: Neuroeconomics, Judgment and Decision Making (pp. 157-180). New York: Psychology Press.

Gladwin, T. E., \& Figner, B. (2019). Trial-to-trial carryover effects on spatial attentional bias. Acta Psychologica, 196, 51-55. https://doi.org/10.1016/J.ACTPSY.2019.04.006

Gladwin, T. E., Figner, B., Crone, E. A., \& Wiers, R. W. (2011). Addiction, adolescence, and the integration of control and motivation. Developmental Cognitive Neuroscience, 1(4), 364-376. https://doi.org/10.1016/j.den.2011.06.008

Gladwin, T. E., Möbius, M., \& Becker, E. S. (2019). Predictive Attentional Bias Modification induces stimulus-evoked attentional bias for threat. Europe's Journal of Psychology, In press.

Gladwin, T. E., Möbius, M., Mcloughlin, S., \& Tyndall, I. (2019). Anticipatory versus reactive spatial attentional bias to threat. British Journal of Psychology, 110(1), 3-14. https://doi.org/10.1111/bjop.12309

Gladwin, T. E., \& Vink, M. (2018). Alcohol-related attentional bias variability and conflicting automatic associations. Journal of Experimental Psychopathology, 9(2). https://doi.org/10.5127/jep.062317

Gray, K. L. H., Adams, W., \& Garner, M. (2010). Preferential processing of fear faces: Emotional content vs. Low-level visual properties. Journal of Vision, 10(7), 610-610. https://doi.org/10.1167/10.7.610

Gray, K. L. H., Adams, W. J., Hedger, N., Newton, K. E., \& Garner, M. (2013). Faces and awareness: Low-level, not emotional factors determine perceptual dominance. Emotion, 13(3), 537-544. https://doi.org/10.1037/a0031403 
Anticipatory attentional bias

Hill, M., \& Duval, E. (2016). Exploring Carry-Over Effects to Elucidate Attention Bias Modification's Mixed Results. Journal of Young Investigators, 31(3), 9-14. https://doi.org/10.22186/jyi.31.3.9-14

Hogarth, L., \& Duka, T. (2006). Human nicotine conditioning requires explicit contingency knowledge: Is addictive behaviour cognitively mediated? Psychopharmacology, 184(3-4), 553-66. https://doi.org/10.1007/s00213-005-0150-0

Hollerman, J. R., Tremblay, L., \& Schultz, W. (2000). Involvement of basal ganglia and orbitofrontal cortex in goal-directed behavior. Progress in Brain Research, 126, 193 215. https://doi.org/10.1016/S0079-6123(00)26015-9

Hommel, B. (2004). Event files: Feature binding in and across perception and action. Trends Cogn Sci, 8(11), 494-500.

Iacoviello, B. M., Wu, G., Abend, R., Murrough, J. W., Feder, A., Fruchter, E., ... Charney, D. S. (2014). Attention bias variability and symptoms of posttraumatic stress disorder. Journal of Traumatic Stress, 27(2), 232-239. https://doi.org/10.1002/jts.21899

Kappenman, E. S., Farrens, J. L., Luck, S. J., \& Proudfit, G. H. (2014). Behavioral and ERP measures of attentional bias to threat in the dot-probe task: Poor reliability and lack of correlation with anxiety. Frontiers in Psychology, 5(DEC), 1368. https://doi.org/10.3389/fpsyg.2014.01368

Korucuoglu, O., Gladwin, T. E., \& Wiers, R. W. (2014). Preparing to approach or avoid alcohol: EEG correlates, and acute alcohol effects. Neuroscience Letters, 559, 199204. https://doi.org/10.1016/j.neulet.2013.12.003

Koster, E. H. W., Crombez, G., Van Damme, S., Verschuere, B., \& De Houwer, J. (2005). Signals for threat modulate attentional capture and holding: Fear-conditioning and extinction during the exogenous cueing task. Cognition \& Emotion, 19(5), 771-780. https://doi.org/10.1080/02699930441000418 
Anticipatory attentional bias

Kruijt, A.-W., Field, A. P., Fox, E., Thompson, E., Reinecke, A., \& Beevers, C. (2016). Capturing Dynamics of Biased Attention: Are New Attention Variability Measures the Way Forward? PLOS ONE, 11(11), e0166600. https://doi.org/10.1371/journal.pone.0166600

Lavender, T., \& Hommel, B. (2007). Affect and action: Towards an event-coding account. Cognition \& Emotion, 21(6), 1270-1296. https://doi.org/10.1080/02699930701438152

Le Pelley, M. E., Vadillo, M., \& Luque, D. (2013). Learned predictiveness influences rapid attentional capture: Evidence from the dot probe task. Journal of Experimental Psychology: Learning, Memory, and Cognition, 39(6), 1888-1900.

MacLeod, C. M., Grafton, B., \& Notebaert, L. (2019). Anxiety-Linked Attentional Bias: Is It Reliable? Annual Review of Clinical Psychology, 15(1), annurev-clinpsy-050718095505. https://doi.org/10.1146/annurev-clinpsy-050718-095505

MacLeod, C. M., Mathews, A., \& Tata, P. (1986). Attentional bias in emotional disorders. Journal of Abnormal Psychology, 95(1), 15-20.

McNally, R. J. (2018, May 21). Attentional bias for threat: Crisis or opportunity? Clinical Psychology Review. https://doi.org/10.1016/j.cpr.2018.05.005

Mogg, K., \& Bradley, B. P. (1999). Orienting of Attention to Threatening Facial Expressions Presented under Conditions of Restricted Awareness. Cognition \& Emotion, 13(6), 713-740. https://doi.org/10.1080/026999399379050

Mogg, K., Holmes, A., Garner, M., \& Bradley, B. P. (2008). Effects of threat cues on attentional shifting, disengagement and response slowing in anxious individuals. Behaviour Research and Therapy, 46(5), 656-667. https://doi.org/10.1016/j.brat.2008.02.011

Monsell, S. (2003). Task switching. Trends in Cognitive Sciences, 7(3), 134-140. 
Anticipatory attentional bias

Noël, X., Colmant, M., Van Der Linden, M., Bechara, A., Bullens, Q., Hanak, C., \& Verbanck, P. (2006). Time course of attention for alcohol cues in abstinent alcoholic patients: The role of initial orienting. Alcoholism, Clinical and Experimental Research, 30(11), 1871-7. https://doi.org/10.1111/j.1530-0277.2006.00224.x

Notebaert, L., Crombez, G., Van Damme, S., De Houwer, J., \& Theeuwes, J. (2011). Signals of threat do not capture, but prioritize, attention: A conditioning approach. Emotion, 11(1), 81-9. https://doi.org/10.1037/a0021286

Pourtois, G., \& Vuilleumier, P. (2006). Dynamics of emotional effects on spatial attention in the human visual cortex. Progress in Brain Research, 156, 67-91. https://doi.org/10.1016/S0079-6123(06)56004-2

Puls, S., \& Rothermund, K. (2018). Attending to emotional expressions: No evidence for automatic capture in the dot-probe task. Cognition and Emotion, 32(3), 450-463. https://doi.org/10.1080/02699931.2017.1314932

Roelfsema, P. R., Engel, A. K., König, P., \& Singer, W. (1997). Visuomotor integration is associated with zero time-lag synchronization among cortical areas. Nature, 385(6612), 157-161. https://doi.org/10.1038/385157a0

Rushworth, M. F. S., Passingham, R. E., \& Nobre, A. C. (2005). Components of attentional set-switching. Experimental Psychology, 52(2), 83-98. https://doi.org/10.1027/16183169.52.2.83

Samejima, K., \& Doya, K. (2007). Multiple representations of belief states and action values in corticobasal ganglia loops. Annals of the New York Academy of Sciences, 1104, 213-228. https://doi.org/10.1196/annals.1390.024

Schmukle, S. C. (2005). Unreliability of the dot probe task. European Journal of Personality, 19(7), 595-605. https://doi.org/10.1002/per.554 
Anticipatory attentional bias

Singer, W., Kreiter, A., Engel, A., Fries, P., Roelfsema, P., \& M, V. (1996). Precise timing of neuronal discharges within and across cortical areas: Implications for synaptic transmission. J Physiol Paris, 90, 221-222.

Slagter, H. A., Alilovic, J., \& Van Gaal, S. (2018). How early does attention modulate visual information processing? The importance of experimental protocol and data analysis approach. Cognitive Neuroscience, 9(1-2), 26-28. https://doi.org/10.1080/17588928.2017.1372405

Swick, D., \& Ashley, V. (2017). Enhanced Attentional Bias Variability in Post-Traumatic Stress Disorder and its Relationship to More General Impairments in Cognitive Control. Scientific Reports, 7(1), 14559. https://doi.org/10.1038/s41598-017-15226-7

The Mathworks. (2015). MATLAB. Natick, Massachusetts: The Mathworks, Inc.

Thoma, P., Soria Bauser, D., \& Suchan, B. (2013). BESST (Bochum Emotional Stimulus Set) - A pilot validation study of a stimulus set containing emotional bodies and faces from frontal and averted views. Psychiatry Research, 209(1), 98-109. https://doi.org/10.1016/j.psychres.2012.11.012

Townshend, J. M., \& Duka, T. (2001). Attentional bias associated with alcohol cues: Differences between heavy and occasional social drinkers. Psychopharmacology, 157(1), 67-74. https://doi.org/10.1007/s002130100764

Townshend, J. M., \& Duka, T. (2007). Avoidance of alcohol-related stimuli in alcoholdependent inpatients. Alcoholism, Clinical and Experimental Research, 31(8), 134957. https://doi.org/10.1111/j.1530-0277.2007.00429.x

Vollstädt-Klein, S., Loeber, S., von der Goltz, C., Mann, K., \& Kiefer, F. (2009). Avoidance of alcohol-related stimuli increases during the early stage of abstinence in alcoholdependent patients. Alcohol and Alcoholism (Oxford, Oxfordshire), 44(5), 458-63. https://doi.org/10.1093/alcalc/agp056 
Anticipatory attentional bias

Waechter, S., Nelson, A. L., Wright, C., Hyatt, A., \& Oakman, J. (2014). Measuring attentional bias to threat: Reliability of dot probe and eye movement indices.

Cognitive Therapy and Research, 38(3), 313-333. https://doi.org/10.1007/s10608$013-9588-2$

Zvielli, A., Bernstein, A., \& Koster, E. H. W. (2014). Dynamics of attentional bias to threat in anxious adults: Bias towards and/or away? PloS One, 9(8), e104025. https://doi.org/10.1371/journal.pone.0104025 
Anticipatory attentional bias

Table 1. Performance data

\begin{tabular}{lllll} 
Probe location & $\begin{array}{l}\text { Previous probe } \\
\text { location }\end{array}$ & CSI $[\mathrm{ms}[$ & RT & Accuracy \\
\hline Neutral & Neutral & 400 & $606(84)$ & $.96(.067)$ \\
Neutral & Neutral & 1000 & $593(80)$ & $.96(.067)$ \\
Neutral & Threat & 400 & $598(82)$ & $.97(.043)$ \\
Neutral & Threat & 1000 & $589(79)$ & $.96(.051)$ \\
Threat & Neutral & 400 & $579(82)$ & $.96(.046)$ \\
Threat & Neutral & 1000 & $577(72)$ & $.97(.047)$ \\
Threat & Threat & 400 & $572(80)$ & $.97(.042)$ \\
Threat & Threat & 1000 & $568(76)$ & $.97(.048)$
\end{tabular}

Note. The Table shows means with SD in brackets of RT and accuracy per condition of the task. The level of probe location (Neutral versus Threat) refers to the stimulus category predicted by the cue at that location. 
Anticipatory attentional bias

Figure 1. Illustration of the cVPT

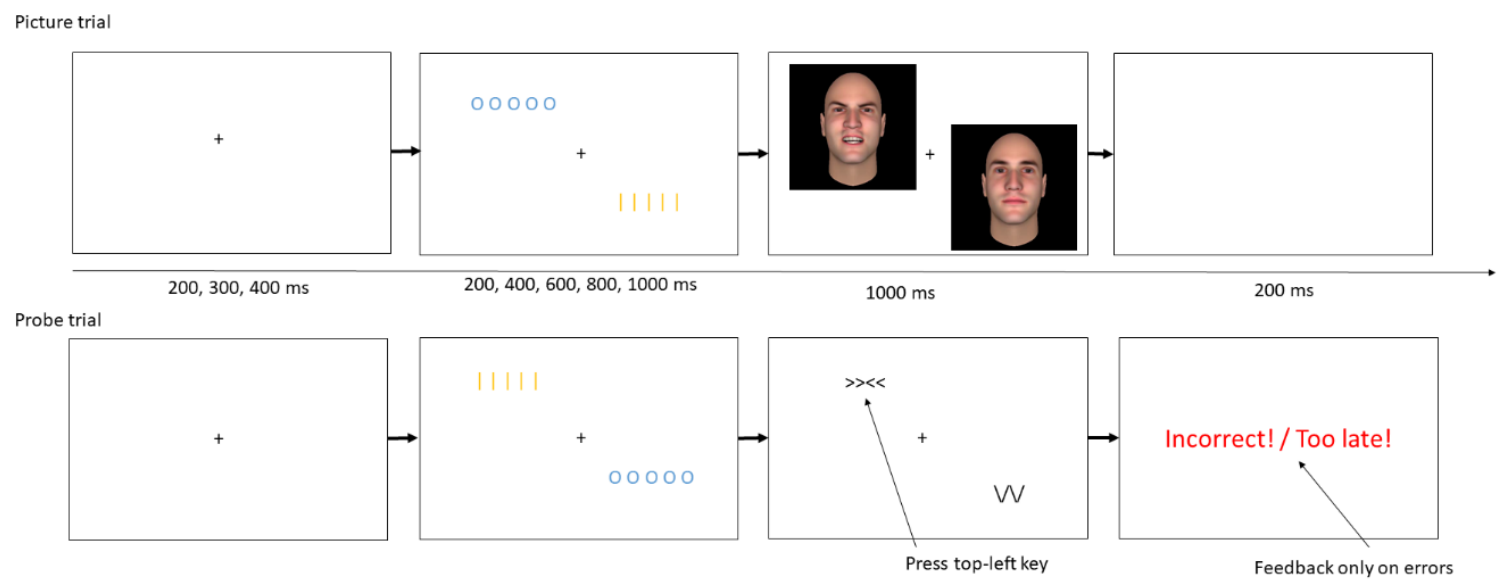

Note. The figure illustrates a Picture and a Probe trial. On Picture trials, no response was required, but pictures of an angry and a neutral face were presented at the locations predicted by the cues. On Probe trials, instead of the pictures a probe appeared requiring the participant to press the response key corresponding to the location of the target stimulus $>><<$. 
Anticipatory attentional bias

Figure 2. Performance data on the cVPT

\section{A. Reaction time}

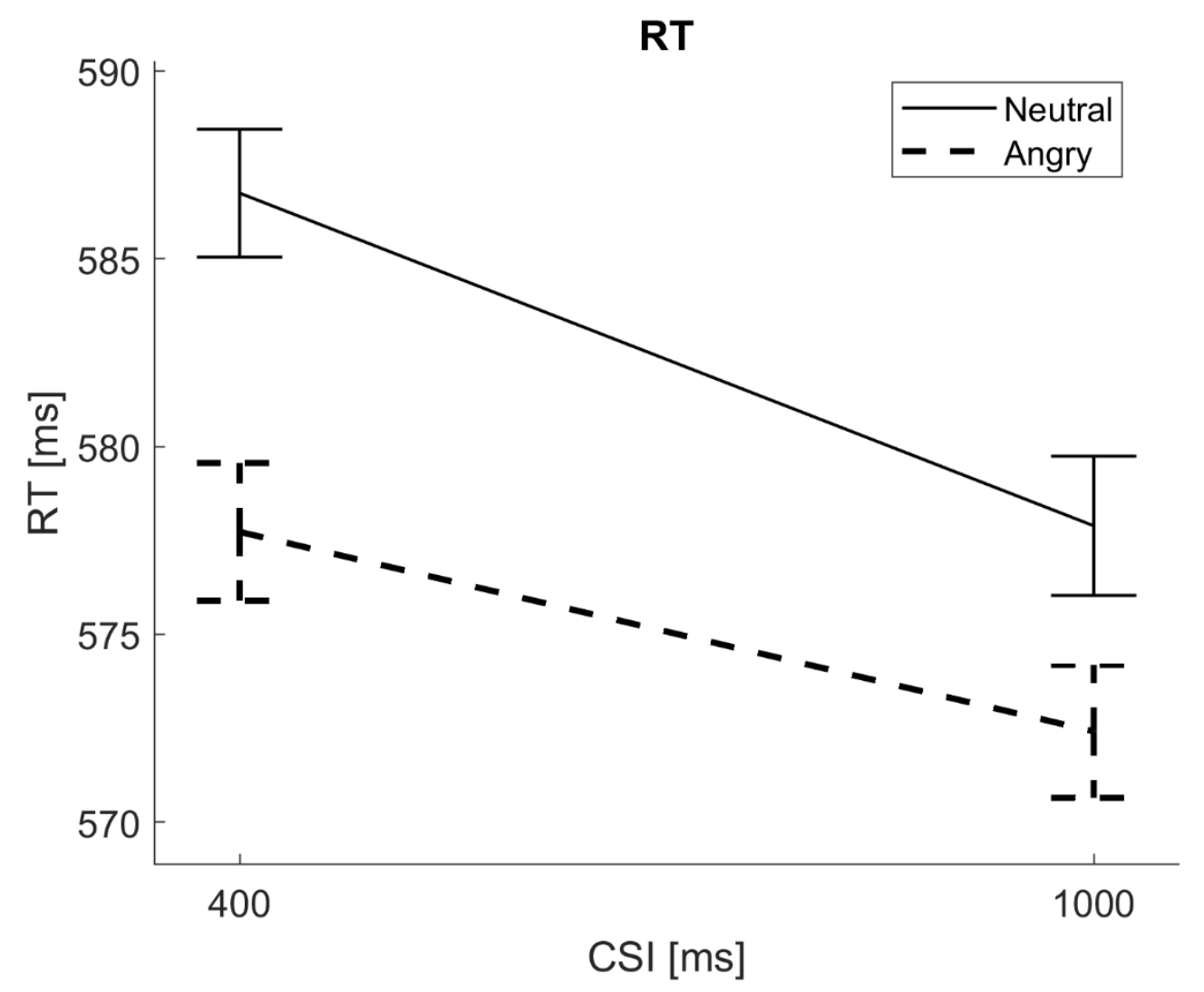

B. Accuracy 
Anticipatory attentional bias

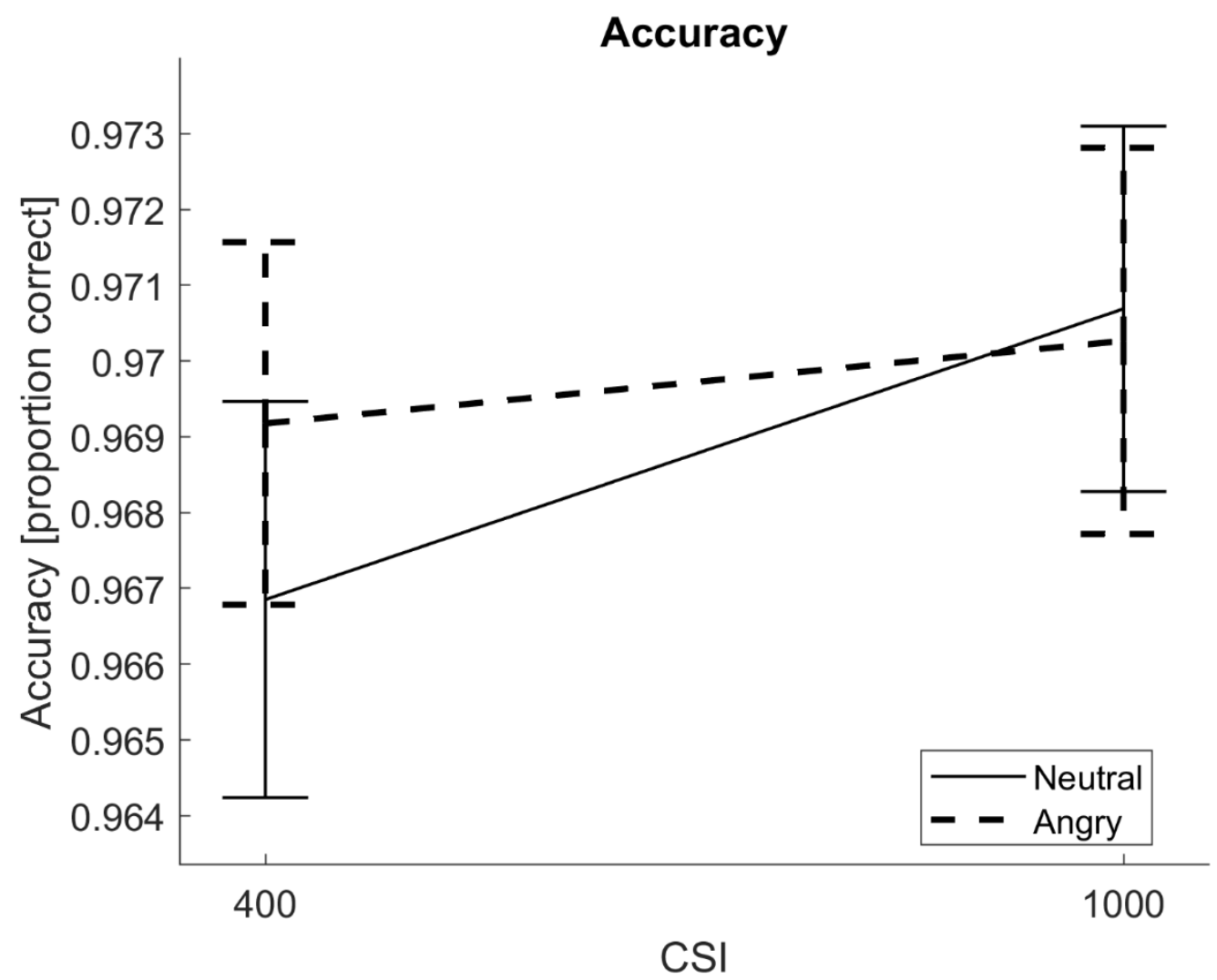

Note. Figure A shows RT data. Figure B shows accuracy data. Errors bars show 1 standard error. Because the analyses involve within-subject effects, standard errors are calculated after removal of subject means. This visualizes the more relevant within-subject noise in condition effects, rather than variation due to differing overall means that is not relevant to the size of within-subject effects. 